\title{
Dispersión de semillas por aves residentes en bosque ribereño urbano del río Torres, San José, Costa Rica
}

\author{
Sergio Gabriel Quesada-Acuña', Carolina Porras Martínez², Oscar Ramírez Alán³ \& Paola Gastezzi-Arias ${ }^{4}$ \\ 1. Laboratorio de Ecología Urbana, Vicerrectoría de Investigación, Universidad Estatal a Distancia, San José, Costa Rica; sgbiotropic@gmail.com \\ 2. Laboratorio Oficial de Análisis de Calidad de Semillas, Centro para Investigaciones en Granos y Semillas, Universidad de Costa Rica; \\ caropm1@yahoo.com \\ 3. Escuela de Ciencias Biológicas de la Universidad Nacional, Heredia, Costa Rica; osoramirez@gmail.com \\ 4. Red Gestión en Conservación de Vida Silvestre y Salud, Vicerrectoría de Investigación, Universidad Estatal a Distancia, San José, Costa Rica; \\ pgastezzi@gmail.com
}

Recibido 20-XI-2017 • Corregido 29-XI-2017 • Aceptado 11-XII-2017

\begin{abstract}
Seed dispersal by resident birds in urban riparian forest, Torres river, San José, Costa Rica. Birds are the main seed dispersers in neotropical ecosystems and it is important to understand their role in the natural regeneration of degraded environments. Here, we analyze dispersion by resident birds in two remnants of riverine forest in the urban watershed of the Torres river, San José, Costa Rica. We visited the sites four times per season, in dry, rainy and transition periods. On each visit we set up standard-size mist nets to capture birds, collect their excreta and extract seeds. In total we captured 168 birds from 23 resident species ( 12 families, five orders). We captured more individuals and species in Dry-Rainy transition, without difference among sites. The Dry-Rainy transition had more total seeds; higher seed capture rate, and a higher seed mean per bird. Resident birds can adapt to different scenarios and their role in seed dispersion should be promoted in urban ecosystems.
\end{abstract}

Key words: Urban birdlife, Urban ecology, Ornithochory, Natural regeneration, Ecological restoration.
RESUMEN: Las aves son el principal dispersor de semillas de los ecosistemas neotropicales y es importante entender su papel en la regeneración natural de los ambientes degradados. En este trabajo, analizamos la dispersión de semillas por aves residentes en dos remanentes de bosque ribereño urbano del río Torres, San José, Costa Rica. Visitamos los sitios cuatro veces por época, en época lluviosa, seca y transición seca-lluviosa. En cada visita colocamos redes de niebla de tamaño estándar para capturar aves, recolectar sus excretas y separar las semillas. En total capturamos 168 aves de 23 especies residentes (12 familias, cinco órdenes). Capturamos más individuos y especies en la transición seca-lluviosa, sin diferencia entre los sitios. La transición seca-lluviosa tuvo mayor cantidad total de semillas, mayor promedio de semillas por individuo y tasa de captura de semillas. Las aves residentes pueden adaptarse a diferentes escenarios y su papel en la dispersión de semillas debe promoverse en los ecosistemas urbanos.

Palabras clave: Avifauna urbana, Ecología urbana, Ornitocoria, Regeneración natural, Restauración ecológica.
Los ecosistemas urbanos ofrecen pocas posibilidades para permitir la regeneración natural porque grandes extensiones de suelo son impermeabilizadas permanentemente con edificaciones (Barrientos \& Monge-Nájera, 2010; Bedoya-Patiño, Estévez-Varón \& Castaño-Villa, 2010; Barrientos, 2013; Monge-Nájera, 2013). En ciertas áreas cercanas a ríos, la topografía y la legislación han obstaculizado el desarrollo habitacional, favoreciendo la subsistencia de fragmentos de bosque ribereño colindantes a cafetales, tacotales o potreros arbolados, los que podrían regenerarse naturalmente si se promoviera la dispersión de semillas (Holl, Loik, Lin \& Samuels, 2000;
Meli, 2003; Acosta-Rojas, Muñoz, Torres \& Corredor, 2012; Estrada \& Sánchez, 2012; Graham \& Page, 2012).

La mayoría de las plantas pioneras neotropicales producen frutos carnosos para que sus semillas sean ingeridas y dispersadas por vertebrados (Bleher \& Bohning-Gaese, 2006; Beckman \& Muller-Landau, 2007), siendo las aves el principal dispersor de semillas por su diversidad, adaptabilidad y capacidad de desplazamiento (Holl et al., 2000; Salvande, Figueroa \& Armesto, 2011; Karubian et al. 2012). La frugivoría de muchas especies de aves está ampliamente documentada (Stiles \& Skutch, 2007; Estrada \& Sánchez, 2012; Garrigues \& Dean, 2014) y 
existen decenas que se han adaptado a vivir en ciudades (Pejchar et al., 2008; Chamberlain, Cannon, Toms, Leech, Hatchwell \& Gaston, 2009; Artavia \& Valle, 2013), donde el estudio de sus funciones ecológicas es importante para desarrollar prácticas que faciliten la regeneración natural de los ambientes degradados (Leighton-Reid, Harris, Martin, Barnett \& Zahawi, 2008; Bedoya-Patiño et al., 2010; Estrada \& Sánchez, 2012; Karubian et al., 2012).

Existen numerosos estudios científicos que analizan la dispersión de semillas por aves (ornitocoria) determinando: la cantidad o la calidad de las semillas dispersadas, pero son escasos los estudios que analizan ambos aspectos simultáneamente (Loiselle \& Blake, 1999; DomínguezDomínguez, Morales-Mávil \& Alba-Landa, 2006; Spiegel \& Nathan, 2007; Traveset, Rodríguez-Pérez \& Pías, 2008; Gasperin \& Pizo, 2012). En Costa Rica, el estudio de la ornitocoria se ha desarrollado principalmente en agropaisajes y pastizales rurales; donde se comprobó que la Iluvia de semillas es escasa, pero aumenta en presencia de aves dispersoras (Barrantes \& Pereira, 2002; Pejchar et al., 2008; Reid, Katsuki \& Holl, 2012).

La regeneración natural de las áreas verdes rodeadas por ciudades es posible en lugares donde coexisten fragmentos de bosque ribereño junto a cafetales, tacotales, potreros arbolados o antiguos cultivos (DiStéfano, Nielsen, Hoomans \& Fournier, 1996; Estrada \& Sánchez, 2012; Barrientos, 2013). Detener la perturbación en estos escenarios permite el restablecimiento de las especies pioneras arbustivas que, al desarrollarse, formarán franjas de vegetación que podrían considerarse verdaderos corredores biológicos interurbanos (Feoli, 2013; MongeNájera, 2013; Gastezzi-Arias, Alvarado-García \& PérezGómez, 2017).

Ante la falta de información sobre la ornitocoria en ecosistemas urbanos, se analizó la dispersión de semillas por aves residentes en remanentes de bosque ribereño en la microcuenca urbana del río Torres, San José, Costa Rica.

\section{MATERIALES Y MÉTODOS}

Sitio de estudio: El río Torres recorre la capital de Costa Rica por $12 \mathrm{~km}$ lineales, abarcando cuatro de los cantones más poblados del país hasta desembocar en el río Virilla (Artavia \& Valle, 2013; Feoli, 2013). La región presenta clima tropical premontano de dos estaciones: seca (diciembre-abril) y lluviosa (mayo-noviembre), con periodos transicionales entre ambas y una precipitación anual entre 2000 y 3 000mm (Barrientos, 2010). A lo largo de la microcuenca seleccionamos dos remanentes de bosque ribereño según cinco condiciones: poco mantenimiento, facilidad de acceso vía terrestre, seguridad para los investigadores, perímetro rodeado por urbanismo y cercanía con potreros, pastizales o jardines. A continuación, describimos ambos sitios:

A. Finca 3, Universidad de Costa Rica, Mercedes de Montes de Oca, San José (09 $56^{\prime} 47^{\prime \prime} \mathrm{N}$ y $\left.84^{\circ} 02^{\prime} 44^{\prime \prime} \mathrm{W}\right)$ : Terreno de aproximadamente 21 ha en la parte media-alta de la microcuenca (1220m.s.n.m.). La mayor parte se compone de jardines con árboles aislados e infraestructura, pero al norte subsisten unas 5ha con remanentes de bosque ribereño sin utilización ni mantenimiento, excepto por algunos senderos recreativos. El bosque ribereño posee un estrato arbustivo y árboles que rondan los $20 \mathrm{~m}$ de altura.

B. Reserva Conejos, Rohrmoser, San José (0956'40" N y $\left.84^{\circ} 06^{\prime} 48^{\prime \prime} \mathrm{W}\right)$ : Terreno de aproximadamente 4 ha en la parte media-baja de la microcuenca (1050m.s.n.m.). Antes del 2005, el terreno era una escombrera cubierta por la gramínea Pennisetum purpureum y posteriormente la Municipalidad de San José (MSJ) coordinó esfuerzos para proteger y aumentar la cobertura del bosque ribereño que actualmente alcanza unas $2 \mathrm{ha}$. El bosque ribereño posee un estrato arbustivo y árboles que rondan los $10 \mathrm{~m}$ de altura.

Diseño de muestreo, captura de aves y recolección de excretas: Visitamos ambos sitios de forma alternada, cuatro veces por época, considerando: la transición seca-lluviosa 2016 (mayo-julio), la época lluviosa 2016 (setiembre-noviembre) y la época seca 2017 (enero-marzo). Tres muestreos en Finca 3 UCR (de época seca) se tuvieron que cancelar por la alteración del sitio.

En cada visita colocamos seis redes de niebla de tamaño estándar (alto 2.60m, largo $12 \mathrm{~m}$, luz de malla $38 \mathrm{~mm}$ ), distribuidas según las rutas de vuelo de las aves entre el bosque ribereño y alrededores (Ralph, Geupel, Pyle, Martin, DeSante \& Milá, 1996). Las redes permanecieron abiertas durante el periodo de mayor actividad de las aves, desde las 06:00 hasta las 12:00 horas (Ralph et al., 1996), para un total acumulado de 756 horas red. Revisamos las redes cada $15 \mathrm{~min}$ para evitar lesiones en las aves, sofoques y pérdida de excretas.

Identificamos las aves hasta nivel de especie utilizando guías especializadas (Stiles \& Skutch, 2007; Garrigues \& Dean, 2014) y las marcamos mediante el corte de una pluma del ala izquierda, para evitar las recapturas. Excluimos a los colibríes (familia Trochilidae) porque no incluyen frutos ni semillas en su dieta, y también a las aves migratorias latitudinales porque no formaban parte de los objetivos del estudio (Stiles \& Skutch, 2007; Estrada \& Sánchez, 2012; Garrigues \& Dean, 2014). 
Al detectar un ave atrapada, colocamos un metro cuadrado de tela en el suelo bajo el ave, para detectar las excretas provocadas por el estrés de la manipulación inicial. Cada ave permaneció en una bolsa de tela limpia e individualizada por un máximo de diez minutos (Moreno-Velásquez, 2010; Hernández-Ladrón de Guevara, Rojas-Soto, López-Barrera, Puebla-Olivares \& Díaz-Castelazo, 2012).

Antes de liberar al ave, revisamos cuidadosamente su plumaje para detectar semillas adheridas: toda semilla que encontramos así se consideró dispersada y fue agregada a las excretas dentro de la bolsa. Al encontrar frutos enteros o fragmentos grandes en las excretas, manipulamos la muestra para obtener sólo las semillas.

Comprobación de germinación: Contamos las semillas de cada excreta y las colocamos en placas Petri esterilizadas de $90 \mathrm{~mm}$ de diámetro, sobre un sustrato de papel filtro cualitativo $\mathrm{N}^{\circ} 101$, humedecido una única vez con $4 \mathrm{~mL}$ de agua destilada y dentro de una cámara de germinación programada: fotoperiodo de 12 horas luz y 12 horas oscuridad, temperatura $30^{\circ} \mathrm{C}$ y humedad relativa $98 \%$.

El recuento de germinación lo realizamos cada 48 horas durante 30 días y consideramos "germinadas"las semiIlas que mostraron una emergencia normal de la radícula (Domínguez-Domínguez et al., 2006; Tenorio-Galindo, Rodríguez-Trejo \& López-Ríos, 2008; Cabrera, Dottori \& Cosa, 2010). Pasados 30 días, evaluamos la viabilidad de las semillas no germinadas utilizando una prueba con tetrazolio, según las recomendaciones generales de International Seed Testing Association (ISTA, 2017).

Análisis estadístico: Para analizar si existían diferencias en la cantidad de individuos o la cantidad de especies capturadas en las distintas épocas; aplicamos una prueba chi cuadrado simple o "bondad de ajuste" para cada caso.

Calculamos una tasa de captura de individuos para cada época y sitio (cantidad de individuos capturados / esfuerzo de muestreo en horas red * 100). De la misma forma, se calculó una tasa de captura de especies para cada época y sitio. Con ambas tasas realizamos pruebas de independencia de chi cuadrado por "tabla de contingencia", para comprobar que las muestras de ambos sitios eran asociables.

Analizamos estadísticos descriptivos y promedios para identificar cuáles especies de aves residentes dispersaron mayor cantidad de semillas y determinar si en alguna época del año se registra un aumento en la dispersión de semillas por aves residentes. También calculamos una tasa de captura de semillas para cada época (cantidad total de semillas / esfuerzo de muestreo en horas red * 100).

\section{RESULTADOS}

Capturamos un total de 168 aves de 23 especies residentes, pertenecientes a 12 familias y cinco órdenes (Apéndice 1). Las familias con mayor número de especies fueron: Passerellidae y Thraupidae ( $n=4$, cada una); seguidas por Troglodytidae ( $n=3$, cada una); tres familias: Columbidae, Icteridae y Tyrannidae ( $n=2$, cada una) y seis familias representadas por una especie (Apéndice 1).

La cantidad de individuos $\left(X^{2}=51,93, \mathrm{gl}=2, \mathrm{p}<0,0001\right)$ $y$ de especies $\left(X^{2}=11,14, g l=2, p=0,004\right)$ capturadas en Finca 3 UCR fue significativamente mayor durante la transición seca-lluviosa (Cuadro 1). Se obtuvo el mismo resultado en Reserva Conejos $\left(X^{2}=42,30, g \mathrm{l}=2, \mathrm{p}<0,0001\right)$, $\left(X^{2}=4,71, \mathrm{gl}=2, \mathrm{p}=0,095\right)$, respectivamente (Cuadro 1$)$.

La tasa de captura de individuos $\left(X^{2}=0,52, \mathrm{gl}=2\right.$, $p=0,77)$ y la tasa de captura de especies $\left(X^{2}=0,13, g l=2\right.$,

\section{CUADRO 1}

Parámetros comparativos en la captura de aves residentes en dos remanentes de bosque ribereño en la microcuenca urbana del río Torres, San José, Costa Rica; durante la transición seca-lluviosa 2016 (T16), época lluviosa 2016 (L16) y época seca 2017 (S17)

\begin{tabular}{|c|c|c|c|c|c|c|}
\hline \multirow{3}{*}{ Parámetros comparativos } & \multicolumn{6}{|c|}{ Sitio de muestreo y Épocas del año } \\
\hline & \multicolumn{3}{|c|}{ Finca 3 UCR } & \multicolumn{3}{|c|}{ Reserva Conejos } \\
\hline & T16 & L16 & S17 & T16 & L16 & S17 \\
\hline Esfuerzo de muestreo en horas red & 144 & 144 & 36 & 144 & 144 & 144 \\
\hline Total de individuos capturados & 55 & 22 & 3 & 58 & 17 & 13 \\
\hline Total de especies capturadas & 14 & 5 & 2 & 16 & 7 & 8 \\
\hline Tasa de captura de individuos & 38,19 & 15,28 & 8,33 & 40,28 & 11,80 & 9,03 \\
\hline Tasa de captura de especies & 9,72 & 3,47 & 5,55 & 11,11 & 4,86 & 5,55 \\
\hline
\end{tabular}


$p=0,94)$ para cada sitio y época respectivamente, no mostraron diferencia estadística (Cuadro 1), de manera que ambos sitios se analizaron en conjunto.

Las únicas especies que dispersaron semillas en todas sus excretas fueron Saltator coerulescens y Saltator maximus (Cuadro 2).

Según la cantidad total de semillas por especie, las especies que dispersaron más fueron: Turdus grayi, Thraupis episcopus, Momotus lessonii, S. coerulescens y Melanerpes hoffmannii ( $\left.X^{2}=2097,7, \mathrm{gl}=4, \mathrm{p}<0,0001\right)$ y según el promedio de semillas por individuo, las especies que dispersaron más fueron: $S$. coerulescens, $M$. hoffmannii, $T$. episcopus, $M$. lessonii y $T$. grayi $\left(X^{2}=14,07, g l=4, p=0,007\right)$. En ambos parámetros se observaron diferencias estadísticas significativas (Cuadro 2).

Obtuvimos 69 excretas con semillas en total y en el $70 \%$ de los casos (48 excretas) las semillas germinaron (Cuadro 3). La prueba bioquímica con tetrazolio aplicada a las semillas no germinadas resultó negativa (semillas no viables).
Según la cantidad total de semillas $\left(X^{2}=2 \quad 389,8\right.$, $\mathrm{gl}=2, \mathrm{p}<0,0001)$, el promedio de semillas por individuo $\left(X^{2}=5,93, g l=2, p=0,051\right)$ y la tasa de captura de semillas $\left(X^{2}=779,36, g l=2, p<0,0001\right)$, las especies de aves residentes dispersaron más durante la transición seca-lluviosa (Cuadro 3).

\section{DISCUSIÓN}

Los resultados de este estudio sugieren que las aves residentes pueden adaptarse a diferentes escenarios urbanos y cumplir en ellos sus funciones ecológicas (Guix, 2007; Bojorges-Baños, 2009; Chamberlain et al., 2009; Fujita \& Koike, 2009; Wenny et al., 2011; Artavia \& Valle, 2013), pues se encontraron similitudes importantes en dos remanentes de bosque ribereño de diferente forma, tamaño, altitud y uso de suelo. Finca 3 UCR conserva un remanente de bosque ribereño que se ha regenerado naturalmente durante varios años; mientras que Reserva Conejos protege un remanente mucho menor

\section{CUADRO 2}

Desempeño anual de las especies de aves residentes que dispersaron semillas en dos remanentes de bosque ribereño en la microcuenca urbana del río Torres, San José, Costa Rica; durante la transición seca-lluviosa 2016 (T16), época lluviosa 2016 (L16) y época seca 2017 (S17)

\begin{tabular}{|c|c|c|c|c|}
\hline Especie & $\begin{array}{l}\text { Excretas con semillas } \\
\text { (Proporción) }\end{array}$ & Total de semillas & $\begin{array}{l}\text { Promedio de semillas } \\
\text { por individuo }\end{array}$ & Época de capturas \\
\hline S. coerulescens & 4 de $4(1,00)$ & 196 & 49,00 & $\mathrm{~T} 16, \mathrm{~S} 17$ \\
\hline M. hoffmannii & 2 de $3(0,66)$ & 144 & 48,00 & T16 \\
\hline T. episcopus & 7 de $10(0,70)$ & 382 & 38,20 & T16 \\
\hline M. lessonii & 6 de $10(0,60)$ & 357 & 35,70 & T16, L16, S17 \\
\hline T. grayi & 44 de $68(0,64)$ & 1383 & 20,33 & T16, L16, S17 \\
\hline M. leucotis & 2 de $13(0,15)$ & 33 & 2,53 & T16, L16, S17 \\
\hline T. melancholicus & 1 de $3(0,33)$ & 3 & 1,00 & $\mathrm{~T} 16$ \\
\hline P. sulphuratus & 2 de $5(0,40)$ & 5 & 1,00 & T16 \\
\hline S. maximus & 1 de $1(1,00)$ & 1 & 1,00 & T16 \\
\hline
\end{tabular}

CUADRO 3

Parámetros comparativos en las semillas dispersadas por aves residentes en dos remanentes de bosque ribereño en la microcuenca urbana del río Torres, San José, Costa Rica; durante la transición seca-lluviosa 2016 (T16), época lluviosa 2016 (L16) y época seca 2017 (S17)

\begin{tabular}{lccc}
\multicolumn{1}{c}{ Parámetros comparativos } & \multicolumn{3}{c}{ Épocas del año } \\
Esfuerzo de muestreo en horas red & T16 & L16 & 180 \\
Excretas con semillas (Proporción) & 288 & 12 de $39(0,31)$ & 4 de $16(0,25)$ \\
Excretas con semillas que germinaron & 53 de $113(0,47)$ & 4 de $12(0,33)$ & 4 de $4(1,00)$ \\
Total de semillas & 40 de $53(0,75)$ & 442 & 92 \\
Promedio de semillas por individuo & 1970 & 11,33 & 5,75 \\
Tasa de captura de semillas & 17,43 & 153,47 & 51,11 \\
\hline
\end{tabular}


que posiblemente sea más vulnerable a alteraciones (Quesada-Acuña et al., 2012).

En ambos sitios, el total de individuos y especies capturadas durante la transición seca-lluviosa superó en más del doble los resultados obtenidos para las otras épocas, lo cual es importante para la dispersión de semillas si se considera que la abundancia de la avifauna (no su diversidad), es el factor que más influye en la riqueza de las semillas dispersadas en agropaisajes rurales (Pejchar et al., 2008). Esta abundancia también coincide con la época de reproducción y migración altitudinal de ciertas especies de aves (Janzen, 1991; Stiles \& Skutch, 2007) y refuerza la importancia de dicho periodo para la regeneración natural (Menacho-Odio \& Sáenz, 2004; Zahawi \& Holl, 2009; Valle, 2013).

La mayoría de especies capturadas pueden considerarse comunes y abundantes en la microcuenca urbana del río Torres y su aporte a la dispersión de semillas puede explicarse por sus respectivas dietas (Stiles \& Skutch, 2007; Pérez-Gómez, Gastezzi-Arias \& Vega-Quesada, 2016).

Las palomas (familia Columbidae), se caracterizan por consumir semillas o frutos que recogen del suelo y son procesados en su molleja muscular, de manera que podrían considerarse depredadoras de semillas o dispersoras ocasionales (Stiles \& Skutch, 2007; Hernández-Ladrón de Guevara et al., 2012). En esta investigación ninguna paloma dispersó semillas, pero en otros escenarios del valle central costarricense se han reportado palomas dispersoras: Columba livia, Zenaida asiatica y Patagioenas flavirostris (Estrada \& Sánchez, 2012); que se observaron durante el estudio.

Los cuclillos (familia Cuculidae), los trepatroncos (familia Furnariidae) y los soterreyes (familia Troglodytidae), son aves estrictamente insectívoras (Stiles \& Skutch, 2007) y era de esperar que dispersaran pocas o ninguna semilla, igual a lo reportado en otros estudios (Leck, 1969; Poulin, Lefebvre \& McNeil, 1994; Rougés \& Blake, 2001; Amico \& Aizen, 2005).

La familia Icteridae, compuesta por aves omnívoras (Stiles \& Skutch, 2007), tampoco dispersó semillas durante el estudio; pero la escasa muestra de individuos impide hacer mayores inferencias sobre su aporte a la dispersión de semillas en ecosistemas urbanos. Es posible que las especies capturadas estén dispersando semillas efectivamente (Poulin et al., 1994) y también se observaron otras especies del grupo con potencial dispersor: Psarocolius montezuma, Dives dives y Sturnella magna (Estrada \& Sánchez, 2012).

Las reinitas (familia Parulidae) son pequeñas aves insectívoras que complementan su dieta con bayas, semillas ariladas y néctar (Stiles \& Skutch, 2007). Se sabe que una amplia mayoría de las especies costarricenses son migratorias (Garrigues \& Dean, 2014) y que aumentan su consumo de frutos hacia el final de la época seca como preparación de su migración hacia el norte (Howe \& DeSteven, 1979; Blake \& Loiselle, 1992). Sin embargo, entre las especies residentes, ningún individuo de Basileuterus rufifrons dispersó semillas, similar a lo reportado en otros estudios (Rougés \& Blake, 2001; Fierro-Calderón, Estela \& Chacón-Ulloa, 2006; MontañoCentellas, 2013).

Los gorriones americanos (familia Passerellidae), se caracterizan por consumir semillas, insectos y frutos, a nivel del suelo o en los arbustos, triturándolos con su pico (Stiles \& Skutch, 2007) y consecuentemente, sólo se obtuvieron semillas viables de dos excretas. Aunque los resultados y otros estudios sugieren que esta familia no dispersa semillas (Rougés \& Blake, 2001; Amico \& Aizen, 2005; Hernández-Ladrón de Guevara et al., 2012; Montaño-Centellas, 2013), el hecho que Melozone leucotis dispersara semillas durante la época de transición seca-lluviosa demuestra que su aporte a la dispersión también puede ser efectivo (Estrada \& Sánchez, 2012).

Las tangaras (familia Thraupidae), pertenecen a un grupo heterogéneo de aves frugívoras con gran potencial para la dispersión de semillas en distintos ecosistemas (Stiles \& Skutch, 2007; Estrada \& Sánchez, 2012), aunque no todos los miembros del grupo cumplen con esta característica. Las especies: Saltator coerulescens, Thraupis episcopus y Saltator maximus, dispersaron semillas en cantidades importantes, principalmente durante la transición seca-lluviosa, de manera similar a lo reportado en otros estudios (Rougés \& Blake, 2001; Guix, 2007; Moreno-Velázquez, 2010; Hernández-Ladrón de Guevara et al., 2012; Montaño-Centellas, 2013). Es importante mencionar que también se observaron otras especies de la familia: Thraupis palmarum y Ramphocelus passerinii, reportadas como dispersoras (Estrada \& Sánchez, 2012).

La familia Tyrannidae, reconocida por capturar insectos al vuelo (Stiles \& Skutch, 2007), aportó algunas semillas durante el periodo de transición seca-lluviosa. Los tiránidos varían su consumo de frutos según la época del año (Leck, 1972; Poulin et al., 1994; Latino \& Beltzer, 1999) y dado que son comunes y abundantes en ambos sitios de estudio (Stiles, 1990), su aporte a la dispersión podría ser considerable, sobre todo por su hábito de percharse en áreas abiertas (Latino \& Beltzer, 1999; Stiles \& Skutch, 2007). De esta familia también se observaron otras especies con potencial para ser dispersoras: Myiozetetes similis y Megarhynchus pitangua (Estrada \& Sánchez, 2012).

Otros estudios han comprobado la importancia de los tiránidos para la dispersión de semillas en 
diferentes escenarios latinoamericanos (Leck, 1969; Howe \& DeSteven, 1979; Howe \& VandeKerckhove, 1979; Barrantes \& Pereira, 2002; Amico \& Aizen, 2005; Guix, 2007; Silva, Figueiredo \& DaSilva, 2008; MorenoVelázquez, 2010; Hernández-Ladrón de Guevara et al., 2012).

La familia Momotidae, con Momotus lessonii como su única especie común y abundante en los ecosistemas urbanos costarricenses (Stiles, 1990; Stiles \& Skutch, 2007) resaltó como un importante dispersor, principalmente durante la transición seca-lluviosa, pero también durante la época seca; a pesar de ser considerada una especie omnívora con preferencia de insectos y vertebrados pequeños (Remsen, Hyde \& Chapman, 1993; Stiles \& Skutch, 2007; Leighton-Reid \& Sánchez-Gutiérrez, 2010; Pesquero, Grassi, Pesquero \& Marques, 2014).

Al parecer, su consumo de frutas es más un suplemento nutricional que puede variar según la época (Remsen et al., 1993), pero su tamaño podría permitirle consumir y dispersar frutos más grandes que otras especies (Estrada \& Sánchez, 2012). En efecto, de todas las aves que dispersaron semillas en este estudio, Momotus lessonii fue la más grande. Al parecer, la familia Momotidae posee gran potencial para la dispersión de semillas (Remsen et al., 1993; Estrada \& Sánchez, 2012), pero los estudios específicos sobre su aporte a la dispersión son sumamente escasos.

Los carpinteros (familia Picidae), representados por la especie Melanerpes hoffmannii, son un grupo de aves principalmente insectívoras (Stiles \& Skutch, 2007), pero se sabe que consumen frutos con regularidad (Estrada \& Sánchez, 2012). Durante este estudio, los individuos dispersaron semillas en la transición seca-lluviosa, pero sólo fue capturado en ese periodo, de manera que no se pudo comparar con las otras épocas.

Se sabe que los carpinteros son dispersores de semillas en muchos escenarios (Howe \& VandeKerckhove, 1979; Guix, 2007); aunque su aporte e importancia aún no ha sido bien definida, probablemente por su baja tasa de captura (Amico \& Aizen, 2005). Del mismo modo, en los muestreos se observaron otros carpinteros con potencial para la dispersión (Estrada \& Sánchez, 2012), como: Dryocopus lineatus y Campephilus guatemalensis.

Finalmente, la especie más capturada fue Turdus grayi (familia Turdidae), aunque una amplia mayoría de sus capturas correspondieron al periodo de transición. El yigüirro, considerado omnívoro (Stiles \& Skutch, 2007), es común y abundante en los ecosistemas urbanos costarricenses (Stiles, 1990) y es de los principales dispersores de semillas en remanentes de bosque ribereño urbano y alrededores (Pejchar et al., 2008; Estrada y Sánchez, 2012).
El yigüirro dispersó más semillas (y aportó más individuos) que las otras ocho especies dispersoras combinadas, resaltando su importancia para la ornitocoria en ecosistemas urbanos (Menacho-Odio \& Sáenz, 2004; Pejchar et al., 2008). El aporte a la dispersión de semillas por los mirlos o zorzales del género Turdus, ha sido ampliamente documentado (Rougés \& Blake, 2001; Barrantes \& Pereira, 2002; Amico \& Aizen, 2005; Guix, 2007; Silva et al., 2008; Salvande et al., 2011, HernándezLadrón de Guevara et al., 2012).

En todos los parámetros comparativos de este estudio se comprobó que la ornitocoria fue mayor durante el periodo de transición seca-lluviosa. Así, si la abundancia de frutos en los remanentes de bosque ribereño urbano, se ve reflejada en el contenido de las excretas y en la tasa de captura de las aves residentes (Blake \& Loiselle, 1991; Loiselle \& Blake, 1994); puede sugerirse que la transición seca-lluviosa debe ser un periodo prioritario al establecer una estrategia de manejo para favorecer la regeneración natural de remanentes de bosque ribereño urbano (Zahawi \& Holl, 2009; Valle, 2013).

Ante la actual fragmentación de los hábitats, las universidades y municipalidades tienen el conocimiento y la responsabilidad (Barrientos \& Monge-Nájera, 2011), de promover la revegetación planificada en la época correcta y con las especies adecuadas para incrementar la oferta de recursos a las aves (Reid, Harris, Martin, Barnett \& Zahawi, 2008; Barrientos \& Monge-Nájera, 2010; Estrada, 2013; Valle, 2013), de tal manera que los remanentes de bosque ribereño urbano se conviertan en una importante fuente de alimento y refugio para las comunidades de aves y éstas a su vez, puedan favorecer la regeneración natural de las áreas verdes aledañas; mejorando el ingreso de semillas, facilitando las etapas de sucesión y promoviendo la biodiversidad dentro de las ciudades (Menacho-Odio \& Sáenz, 2004; Morales, 2009; Cole, Holl, Keene \& Zahawi, 2011; Estrada \& Sánchez, 2012).

\section{AGRADECIMIENTOS}

Agradezco a Gabriela Pérez, Julián Monge, Ligia Bermúdez, Mónica González y Zaidett Barrientos, por sus valiosas sugerencias para mejorar el manuscrito. Al Proyecto "Vulnerabilidad Biofísica de la Microcuenca del Río Torres, San José, Costa Rica", de la Vicerrectoría de Investigación, UNED. AI Laboratorio Oficial de Análisis de Calidad de Semillas del Centro para Investigaciones en Granos y Semillas (CIGRAS), UCR.

\section{REFERENCIAS}


Acosta-Rojas, D. C., Muñoz, M. C., Torres, A. M., \& Corredor, G. (2012). Dieta y dispersión de semillas: ¿Afecta la guacharaca colombiana (Ortalis columbiana) la germinación de las semillas consumidas? Ornitología Neotropical, 23, 439-453.

Amico, G. C. \& Aizen, M. A. (2005). Dispersión de semillas por aves en un bosque templado de Sudamérica austral: ¿Quién dispersa a quién? Ecología Austral, 15, 89-100.

Artavia, R. \& Valle, D. (2013). Diagnóstico preliminar de avifauna para instaurar el corredor biológico interurbano río Torres en el cantón San José. Ambientico, 232-233, 56-63.

Barrantes, G. \& Pereira, A. (2002). Seed dissemination by frugivorous birds from forest fragments to adjacent pastures on the western slope of Volcán Barva, Costa Rica. Biología Tropical, 50(2), 569-575.

Barrientos, Z. (2010). Contaminación atmosférica en la Meseta Central de Costa Rica. Biocenosis, 23(1), 50-54.

Barrientos, Z. (2013). ¿Cómo restaurar zonas verdes en ciudades tropicales? Ambientico, 232-233, 81-87.

Barrientos, Z. \& Monge-Nájera, J. (2010). Restauración ecológica en la meseta central de Costa Rica. Biocenosis, 23(2), 20-25.

Barrientos, Z. \& Monge-Nájera, J. (2011). Bioética y biodiversidad en los ecosistemas urbanos. Biocenosis, 24(1-2), 72-80.

Beckman, N. G. \& Muller-Landau, H. C. (2007). Differential effects of hunting on pre-dispersal seed predation and primary and secondary seed removal of two neotropical tree species. Biotropica, 39(3), 328-339. doi: 10.1111/j.1744-7429-2007-00273-x

Bedoya-Patiño, J. G., Estévez-Varón, J. V., \& Castaño-Villa, G. J. (2010). Banco de semillas del suelo y su papel en la recuperación de los bosques tropicales. Boletín Científico Museo Historia Natural, 14(2), 77-91.

Blake, J. G. \& Loiselle, B. A. (1991). Variation in resource abundance affects capture rates of birds in three lowland habitats in Costa Rica. The Auk, 108, 114-130.

Blake, J. G. \& Loiselle, B. A. (1992). Fruits in the diets of neotropical migrant birds in Costa Rica. Biotropica, 24(2a), 200-210.

Bleher, B. \& Bohning-Gaese, K. (2006). The role of birds in seed dispersal and its consequences for forest ecosystems. Acta Zoologica Sinica, 52, 116-119.

Bojorges-Baños, J. C. (2009). Amenazando la biodiversidad: urbanización y sus efectos en la avifauna. Ciencia y Mar, 13(39), 61-65.

Cabrera, V. A., Dottori, N., \& Cosa, M. T. (2010). Germinación, éxito reproductivo y fenología de Solanum chenopodioides (Solanaceae). Boletín de la Sociedad Argentina de Botánica, 45(1-2), 73-80.

Chamberlain, D. E., Cannon, A. R., Toms, M. P., Leech, D. I., Hatchwell, B. J., \& Gaston, K. J. (2009). Avian productivity in urban landscapes: a review and meta-analysis. Ibis, $151,1-18$.

Cole, R. J., Holl, K. D., Keene, C. L., \& Zahawi, R. A. (2011). Direct seeding of late successional trees to restore tropical montane forest. Forest Ecology and Management, 261, 1590-1597. doi: 10.1016/j.foreco.2010.06.038

DiStéfano, J. F., Nielsen, V., Hoomans, J., \& Fournier, L. A. (1996). Regeneración de la vegetación arbórea en una pequeña reserve forestal urbana del nivel premontano húmedo, Costa Rica. Biología Tropical, 44(2), 575-580.

Domínguez-Domínguez, L. E., Morales-Mávil, J. E., \& AlbaLanda, J. (2006). Germinación de semillas de Ficus insípida (Moraceae) defecadas por tucanes (Ramphastos sulfuratus) y monos araña (Ateles geoffroyi). Biología Tropical, 54(2), 387-394.

Estrada, A. (2013). Importancia de los diferentes tipos de cobertura arbórea para la recuperación de la biodiversidad en ambientes urbanos. Ambientico, 232-233, 13-19.

Estrada, A. \& Sánchez, J. E. (2012). Árboles y arbustos de importancia para las aves del Valle central de Costa Rica. Santo Domingo de Heredia: Instituto Nacional de Biodiversidad INBio.

Feoli, S. (2013). Corredor biológico interurbano del río Torres y corredores biológicos en general. Ambientico, 232-233, 51-55.

Fierro-Calderón, K., Estela, F. A., \& Chacón-Ulloa, P. (2006). Observaciones sobre la dieta de algunas aves de la cordillera oriental de Colombia a partir del análisis de contenidos estomacales. Ornitología colombiana, 4, 6-15.

Fujita, M. \& Koike, F. (2009). Landscape effects on ecosystems: birds as active vectors of nutrient transport to fragmented urban forest versus forest-dominated landscapes. Ecosystems, 12, 391-400. Doi: 10.1007/ s10021-009-9230-z.

Garrigues, R. \& Dean, R. (2014). The Birds of Costa Rica: A field guide. New York: Cornell University Press.

Gasperin, G. \& Pizo, M. A. (2012). Passage time of seeds through the guts of frugivorous birds, a first assessment in Brazil. Brasileira de Ornitologia, 20(1), 48-51.

Gastezzi-Arias, P., Alvarado-García, V., \& Pérez-Gómez, G. (2017). La importancia de los ríos como corredores interurbanos. Biocenosis, 31(1-2), 39-45.

Graham, L. L. B. \& Page, S. E. (2012). Artificial bird perches for the regeneration of degraded tropical peat swamp forest: a restoration tool with limited potential. Restoration Ecology, 20(5), 631-637. doi: 10.1111/j.1526-100x-2011-00805-x

Guix, J. C. (2007). The role of alien plants in the composition of fruit-eating bird assemblages in Brazilian urban ecosystems. Orsis, 22, 87-104.

Hernández-Ladrón de Guevara, I., Rojas-Soto, O. R., LópezBarrera, F., Puebla-Olivares, F., \& Díaz-Castelazo, C. (2012). Dispersión de semillas por aves en un paisaje 
de bosque mesófilo en el centro de Veracruz, México: su papel en la restauración pasiva. Chilena de Historia Natural, 85, 89-100.

Holl, K. D., Loik, M. E., Lin, E. H. V., \& Samuels, I. A. (2000). Tropical montane forest restoration in Costa Rica: Overcoming barriers to dispersal and establishment. Restoration Ecology, 8(4), 339-349.

Howe, H. F. \& DeSteven, D. (1979). Fruit production, migrant bird visitation and seed dispersal of Guarea glabra in Panama. Oecologia, 39, 185-196.

Howe, H. F. \& VandeKerckhove, G. A. (1979). Fecundity and seed dispersal of a tropical tree. Ecology, 60(1), 180-189.

International Seed Testing Association, ISTA. (2017). International rules for seed testing, vol. 2017. Bassersdorf, Suiza: ISTA.

Janzen, D. H. (1991). Historia natural de Costa Rica. San José, Costa Rica: Editorial de la Universidad de Costa Rica.

Karubian, J., Browne, L., Bosque, C., Carlo, T., Galetti, M., Loiselle, B. A., Blake, J. G., Cabrera, D., Duraes, R., Labecca, F. M., Holbrook, K. M., Holland, R., Jetz, W., Kummeth, F., Olivo, J., Ottewell, K., Papadakis, G., Rivas, G., Steiger, S., Voirin, B., \& Wikelski, M. (2012). Seed dispersal by Neotropical birds: emerging patterns and underlying processes. Ornitología Neotropical, 23, 9-24.

Latino, S. \& Beltzer, A. (1999). Ecología trófica del benteveo Pitangus Sulphuratus (Aves: Tyrannidae) en el valle de inundación del río Paraná, Argentina. Orsis, 14, 69-78.

Leck, C. F. (1969). Observations of birds exploiting a Central American fruit tree. Wilson bulletin, 81(3), 264-269.

Leck, C. F. (1972). Seasonal changes in feeding pressures of fruit and nectar eating birds in Panamá. Condor, 74, 54-60.

Leighton-Reid, J., Harris, J. B. C., Martin, L. J., Barnett, J. R., \&Zahawi, R. A. (2008). Distribution and abundance of nearctic-neotropical songbird migrants in a forest restoration site in southern Costa Rica. Journal of Tropical Ecology, 24, 685-688. Doi: 10.1017/S.0266-4674-0800-5415

Leighton-Reid, J. \& Sánchez-Gutiérrez, A. (2010). Observaciones de dos nuevas presas de vertebrados para el momoto común (Momotus momota). Zeledonia, 14(2), 68-72.

Loiselle, B. A. \& Blake, J. G. (1994). Annual variation in birds and plants of a tropical second-growth woodland. Condor, 96, 368-380.

Loiselle, B. A. \& Blake, J. G. (1999). Dispersal of melastome seeds by fruit-eating birds of tropical forest understory. Ecology, 80(1), 330-336. doi: 10.2307/177-001

Meli, P. (2003). Restauración ecológica de bosques tropicales: veinte años de investigación académica. Interciencia, 28(10), 581-589.

Menacho-Odio, R. M. \& Sáenz, J. C. (2004). Monitoreo de la avifauna en fincas con sistemas de producción silvopastoril del cantón de Esparza, Costa Rica. Zeledonia, 8(2), 2-6.
Monge-Nájera, J. (2013). Potencial de las capitales provinciales de Costa Rica para albergar corredores biológicos urbanos. Ambientico, 232-233, 75-79.

Montaño-Centellas, F. A. (2013). Does habitat specificity by frugivorous birds result in uneven seed rain within Bolivian mixed plantations. Ecología Austral, 23, 55-61.

Morales, C. O. (2009). Caracterización florística y estructural de tres fragmentos boscosos secundarios en Cartago, Costa Rica. Biología Tropical, 57(1), 69-82.

Moreno-Velázquez, J. S. (2010). Aves dispersoras de semillas en un remanente de bosque seco tropical en la finca BetanciGuacamayas (Córdoba). (Tesis inédita de licenciatura), Pontificia Universidad Javeriana, Bogotá, Colombia.

Pejchar, L., Pringle, R. M., Ranganathan, J., Zook, J. R., Durán, G., Oviedo, F., \& Daily, G. C. (2008). Birds as agents of seed dispersal in a human-dominated landscape of southern Costa Rica. Biological Conservation, 141, 536-544. doi: 10.1016/j.bio.con-2007-11008

Pérez-Gómez, G., Gastezzi-Arias, P., \& Vega-Quesada, A. (2016). Avifauna poco frecuente en la microcuenca del río Torres, San José, Costa Rica. Zeledonia, 20(2), 20-27.

Pesquero, M. A., Grassi, A., Pesquero, M. F., \& Marques, H. (2014). Feeding of nestlings of the Amazonian motmot (Momotus momota) in southern Goiás, Brazil. Brasileira de Ornitologia, 22(3), 288-291.

Poulin, B., Lefebvre, G., \& McNeil, R. (1994). Diets of land birds from northeastern Venezuela. Condor, 96, 354-367.

Quesada-Acuña, S. G., Valdelomar, V., Arrieta, C., Ruiz, G., Matarrita-Herrera, M., Araya, J. F., \& Sandoval, I. (2012). Caracterización del paisaje y análisis del límite oeste del Parque Nacional Carara, Costa Rica: implicaciones para la conservación de la biodiversidad. Brenesia, 78, 6-11.

Ralph, C. J., Geupel, G. R., Pyle, P., Martin, T. E., DeSante, D. F., \& Milá, B. (1996). Manual de métodos de campo para el monitoreo de aves terrestres. (General Technical Report PSWGTR-159). California: Forest Service.

Reid, J. L., Harris, J. B. C., Martin, L. J., Barnett, J. R., \& Zahawi, R. A. (2008). Distribution and abundance of Nearcticneotropical songbird migrants in a forest restoration site in southern Costa Rica. Tropical Ecology, 24, 685-688. Doi: 10.1017/s.0266-4674-0800-5415

Reid, J. L., Katsuki, K. N., \& Holl, K. D. (2012). Do birds bias measurements of seed rain? Tropical Ecology, 28, 421-422. doi: $10.1017 /$ s.0266-4674-1200-0247

Remsen, J. V., Hyde, M. A., \& Chapman, A. (1993). The diets of neotropical trogons, motmots, barbets and toucans. Condor, 95, 178-192.

Rougés, M. \& Blake, J. G. (2001). Tasas de captura y dietas de aves del sotobosque en el Parque Biológico Sierra de San Javier, Tucumán. Hornero, 16(1), 7-15.

Salvande, M., Figueroa, J. A., \& Armesto, J. J. (2011). Componente cuantitativo de la efectividad de dispersión de semillas 
por aves en el bosque templado de Chiloé, Chile. Bosque, 32(1), 39-45.

Silva, I. A., Figueiredo, R. A., \& DaSilva, D. M. (2008). Feeding visit time of fruit-eating birds in Cerrado plants: revisiting the predation risk model. Brasileira de Zoologia, 25(4), 682-688.

Spiegel, O. \& Nathan, R. (2007). Incorporating dispersal distance into the disperser effectiveness framework: frugivorous birds provide complementary dispersal to plants in a patchy environment. Ecology Letters, 10, 718-728. doi: 10.1111/j.1461-0248-2007-01062x

Stiles, F. G. (1990). La avifauna de la Universidad de Costa Rica y sus alrededores a través de veinte años (1968-1989). Biología Tropical, 38(2B), 361-381.

Stiles, F. G. \& Skutch, A. F. (2007). Guía de aves de Costa Rica. Santo Domingo de Heredia: Instituto Nacional de Biodiversidad INBio.
Tenorio-Galindo, G., Rodríguez-Trejo, D. A., \& López-Ríos, G. (2008). Efecto del tamaño y color de la semilla en la germinación de Cecropia obtusifolia Bertol (Cecropiaceae). Agrociencia, 42(5), 585-593.

Traveset, A., Rodríguez-Pérez, J., \& Pías, B. (2008). Seed trait changes in dispersers' guts and consequences for germination and seedling growth. Ecology, 89, 95-106. doi: 10.1890/07-00941

Valle, D. (2013). Repoblamiento del bosque urbano en el cantón San José. Ambientico, 232-233, 40-45.

Wenny, D. G., DeVault, T. L., Johnson, M. D., Kelly, D., Sekercioglu, C. H., Tomback, D. F., \& Whelan, C. J. (2011). The need to quantify ecosystem services provided by birds. The Auk, 128(1), 1-14. doi: 10.1525/auk.2011.10248.

Zahawi, R. A. \& Holl, K. D. (2009). Comparing the performance of tree stakes and seedlings to restore abandoned tropical pastures. Restoration Ecology, 17(6), 854-864. doi: 10.1111/j.1526100x-2008-00423-x

\section{APÉNDICE 1}

Especies de aves residentes, cantidad de individuos capturados y su dispersión de semillas en dos remanentes de bosque ribereño en la microcuenca urbana del río Torres, San José, Costa Rica; durante la transición seca-lluviosa 2016 (T16), época lluviosa 2016 (L16) y época seca 2017 (S17)

\begin{tabular}{|c|c|c|c|c|c|c|c|}
\hline \multirow{2}{*}{ Orden } & \multirow{2}{*}{ Familia } & \multirow{2}{*}{ Especie } & \multirow{2}{*}{ Dispersó semillas } & \multicolumn{4}{|c|}{ Individuos capturados por época del año } \\
\hline & & & & T16 & L16 & S17 & Total \\
\hline \multirow[t]{2}{*}{ Columbiformes } & \multirow[t]{2}{*}{ Columbidae } & Columbina inca & No & 0 & 0 & 2 & 2 \\
\hline & & Leptotila verreauxi & No & 3 & 1 & 2 & 6 \\
\hline Coraciiformes & Momotidae & Momotus lessonii & $\mathrm{Si}$ & 6 & 2 & 2 & 10 \\
\hline Cuculiformes & Cuculidae & Piaya cayana & No & 1 & 0 & 0 & 1 \\
\hline \multirow[t]{18}{*}{ Passeriformes } & Furnariidae & Thripadectes rufobrunneus & No & 1 & 0 & 0 & 1 \\
\hline & \multirow{2}{*}{ Icteridae } & Molothrus aeneus & No & 1 & 0 & 0 & 1 \\
\hline & & Quiscalus mexicanus & No & 1 & 0 & 0 & 1 \\
\hline & Parulidae & Basileuterus rufifrons & No & 6 & 2 & 2 & 10 \\
\hline & \multirow[t]{4}{*}{ Passerellidae } & Atlapetes albinucha & No & 1 & 0 & 0 & 1 \\
\hline & & Melozone cabanisi & No & 0 & 1 & 0 & 1 \\
\hline & & Melozone leucotis & $\mathrm{Si}$ & 6 & 6 & 1 & 13 \\
\hline & & Zonotrichia capensis & No & 7 & 0 & 0 & 7 \\
\hline & \multirow[t]{4}{*}{ Thraupidae } & Sporophila corvina & No & 2 & 1 & 2 & 5 \\
\hline & & Saltator coerulescens & $\mathrm{Si}$ & 3 & 0 & 1 & 4 \\
\hline & & Saltator maximus & $\mathrm{Si}$ & 1 & 0 & 0 & 1 \\
\hline & & Thraupis episcopus & $\mathrm{Si}$ & 10 & 0 & 0 & 10 \\
\hline & \multirow[t]{3}{*}{ Troglodytidae } & Cantorchilus modestus & No & 3 & 6 & 0 & 9 \\
\hline & & Thryophilus rufalbus & No & 0 & 1 & 1 & 2 \\
\hline & & Troglodytes aedon & No & 4 & 0 & 0 & 4 \\
\hline & Turdidae & Turdus grayi & $\mathrm{Si}$ & 46 & 19 & 3 & 68 \\
\hline & \multirow[t]{2}{*}{ Tyrannidae } & Pitangus sulphuratus & $\mathrm{Si}$ & 5 & 0 & 0 & 5 \\
\hline & & Tyrannus melancholicus & $\mathrm{Si}$ & 3 & 0 & 0 & 3 \\
\hline Piciformes & Picidae & Melanerpes hoffmanni & $\mathrm{Si}$ & 3 & 0 & 0 & 3 \\
\hline
\end{tabular}

